\title{
On Similarity and Reducing Subspaces of the $n$-Shift plus Certain Weighted Volterra Operator
}

\author{
Yucheng Li, Hao Chen, and Wenhua Lan \\ Department of Mathematics, Hebei Normal University, Hebei Province Key Laboratory of \\ Computational Mathematics and Applications, Shijiazhuang 050024, China
}

Correspondence should be addressed to Yucheng Li; liyucheng@hebtu.edu.cn

Received 15 February 2017; Accepted 30 March 2017; Published 13 April 2017

Academic Editor: Young Joo Lee

Copyright ( 2017 Yucheng Li et al. This is an open access article distributed under the Creative Commons Attribution License, which permits unrestricted use, distribution, and reproduction in any medium, provided the original work is properly cited.

Let $g(z)$ be an $n$-degree polynomial $(n \geq 2)$. Inspired by Sarason's result, we introduce the operator $T_{1}$ defined by the multiplication operator $M_{g}$ plus the weighted Volterra operator $V_{g}$ on the Bergman space. We show that the operator $T_{1}$ is similar to $M_{g}$ on some Hilbert space $S_{g}^{2}(\mathbb{D})$. Then for $g(z)=z^{n}$, by using matrix manipulations, the reducing subspaces of the corresponding operator $T_{2}$ on the Bergman space are characterized.

\section{Introduction}

The invariant subspace and reducing subspace problems are interesting and important themes in operator theory. The conjecture is that every bounded linear operator $T$ on a separable Hilbert space $H$ has a nontrivial closed invariant subspace. A closed linear nontrivial subspace $X$ of $H$ is called an invariant subspace for $T$ if $X$ is different from $\{0\}$ and $H$ such that $T X \subset X$. If $X$ and $X^{\perp}$ are both invariant subspaces for $T$, then $X$ is said to be a reducing subspace for $T$. The invariant subspace and reducing subspace problems on the Hardy space and the Bergman space have been studied extensively in the literature. We mention here the papers [1-13] and the books [14-17] which include a lot of the information on the corresponding operator theory.

Let $\mathbb{D}$ be the unit disk in the complex plane $\mathbb{C}$, and let $L_{a}^{2}(\mathbb{D})$ denote the Bergman space of analytic functions which belongs to $L^{2}(\mathbb{D})$, where $L^{2}(\mathbb{D})$ is the space of square integrable functions on $\mathbb{D}$. It is well known that $L_{a}^{2}(\mathbb{D})$ is a Hilbert space. If $f \in L_{a}^{2}(\mathbb{D})$, then

$$
\|f\|_{2}^{2}=\int_{\mathbb{D}}|f(z)|^{2} d A(z)
$$

where $d A$ is the normalized area measure on $\mathbb{D}$, and

$$
d A(z)=\frac{1}{\pi} d x d y=\frac{r}{\pi} d r d \theta .
$$

For $f, g \in L_{a}^{2}(\mathbb{D})$, let

$$
\begin{aligned}
& f(z)=\sum_{k=0}^{\infty} a_{k} z^{k}, \\
& g(z)=\sum_{k=0}^{\infty} b_{k} z^{k},
\end{aligned}
$$

and then the inner product of $f$ and $g$ is defined by

$$
\langle f, g\rangle=\int_{\mathbb{D}} f(z) \overline{g(z)} d A(z)=\sum_{k=0}^{\infty} \frac{a_{k} \bar{b}_{k}}{k+1} .
$$

In this inner product, $L_{a}^{2}(\mathbb{D})$ has an orthonormal basis $\left\{e_{k}\right\}_{k=0}^{\infty}$, where

$$
e_{k}(z)=\sqrt{k+1} z^{k}, \quad(k=0,1, \ldots) .
$$

Let $H^{\infty}(\mathbb{D})$ denote the algebra of bounded analytic functions on $\mathbb{D}$. For $\varphi \in H^{\infty}(\mathbb{D}), M_{\varphi}$ is an analytic multiplication operator on the Bergman space defined by

$$
M_{\varphi} f=\varphi f, \text { for } f \in L_{a}^{2}(\mathbb{D}) .
$$

$M_{\varphi}$ is a bounded linear operator on $L_{a}^{2}(\mathbb{D})$ with

$$
\left\|M_{\varphi}\right\|=\|\varphi\|_{\infty}=\sup \{|\varphi(z)|: z \in \mathbb{D}\} .
$$


Over the years it has been shown that many familiar classes of operators do have invariant subspaces. The lattice of shift operator acting on the Hardy space is completely described by Beurling's Theorem [4]. Sarason (see [11]) characterized all closed invariant subspaces of the Volterra operator

$$
\begin{aligned}
& (V f)(x)=\int_{0}^{x} f(y) d y \\
& \quad \text { for } f \in L^{2}(0,1), 0<y \leq x<1 .
\end{aligned}
$$

In [1], Aleman characterized boundedness and compactness of the integral operator

$$
T_{g} f(z)=\int_{0}^{z} g^{\prime}(w) f(w) d w
$$

between Hardy space $H^{p}$ and $H^{q}$ for $p, q>0$. In [2], using Beurling's Theorem, Aleman and Korenblum studied the complex Volterra operator in the Hardy space $H^{2}(\mathbb{D})$ defined by

$$
(V f)(z)=\int_{0}^{z} f(w) d w .
$$

Then they characterized the lattice of closed invariant subspaces of $V$. Sarason (see [11]) studied the lattice of closed invariant subspaces of $M_{x}+V$ acting on $L^{2}(0,1)$. MontesRodriguez et al. (see [9]) and Cowen et al. (see [5]) used the idea of Sarason to study the invariant subspaces of certain classes of composition operators on the Hardy space. Following Sarason's work, Čučković and Paudyal (see [6]) characterized the lattice of closed invariant subspaces of the shift plus complex Volterra operator on the Hardy space. In their paper, the operator $T$ is defined by

$$
(T f)(z)=z f(z)+\int_{0}^{z} f(w) d w,
$$

$$
\text { for } f \in H^{2}(\mathbb{D}), z \in \mathbb{D} \text {. }
$$

Ball (see [3]) and Nordgren (see [10]) studied the problem of determining the reducing subspaces for an analytic Toeplitz operator on the Hardy space. In [12], Stessin and Zhu gave a complete description of the weighted unilateral shift operator of finite multiplicity on some Hilbert spaces type I and type II. In [13], Zhu described the properties of the commutant of analytic Toeplitz operators with inner function symbols on the Hardy space and the Bergman space and characterized the reducing subspaces of a class of multiplication operators. In 2011, Douglas and Kim in [7] studied the reducing subspaces for an analytic multiplication operator $M_{z^{n}}$ on the Bergman space $A_{\alpha}^{2}\left(A_{r}\right)$ of the annulus $A_{r}$.

Based on the above works, for an $n$-degree polynomial $g(z)(n \geq 2)$, we introduce the operator $T_{1}$ defined by the multiplication operator $M_{g}$ plus the weighted Volterra operator $V_{g}$ on the Bergman space. We show that the operator $T_{1}$ is similar to $M_{g}$ on some Hilbert space $S_{g}^{2}(\mathbb{D})$. Then for $g(z)=z^{n}$, by using matrix manipulations, the reducing subspaces of corresponding operator $T_{2}$ on the Bergman space are characterized.

\section{The Similarity of the Operator $T_{1}$}

For an $n$-degree polynomial $g(z)(n \geq 2)$, the operator $T_{1}$ is defined by

$$
\begin{aligned}
\left(T_{1} f\right)(z) & =M_{g(z)} f(z)+\left(V_{g} f\right)(z) \\
& =M_{g(z)} f(z)+\int_{0}^{z} g^{\prime}(w) f(w) d w \\
& \text { for } f \in L_{a}^{2}(\mathbb{D}) .
\end{aligned}
$$
by

To prove our result, we introduce the space $S_{g}^{2}(\mathbb{D})$ defined

$$
S_{g}^{2}(\mathbb{D})=\left\{h \in H(\mathbb{D}): h(0)=0, \frac{D h}{g^{\prime}} \in L_{a}^{2}(\mathbb{D})\right\},
$$

where $H(\mathbb{D})$ is the space of holomorphic functions on the unit disk, and $D=d / d z$ is the differentiation operator. From the definition of $S_{g}^{2}(\mathbb{D})$, for $h \in S_{g}^{2}(\mathbb{D})$, we have

$$
\begin{aligned}
\int_{\mathbb{D}}|(D h)(z)|^{2} d A(z) & =\int_{\mathbb{D}}\left|g^{\prime}(z) \frac{D h(z)}{g^{\prime}(z)}\right|^{2} d A(z) \\
& \leq\left\|g^{\prime}(z)\right\|_{\infty}^{2}\left\|\frac{D h(z)}{g^{\prime}(z)}\right\|_{2}^{2} .
\end{aligned}
$$

So $D h(z) \in L_{a}^{2}(\mathbb{D})$. It can be shown that, for any holomorphic function $h$ with $h(0)=0$ and $D h(z) \in L_{a}^{2}(\mathbb{D})$, then $\|h\|_{2}^{2} \leq$ $\|D h\|_{2}^{2}$. So the norm of $S_{g}^{2}(\mathbb{D})$ is defined by

$$
\|h\|_{S_{g}^{2}(\mathbb{D})}^{2}=\|h\|_{2}^{2}+\|D h\|_{2}^{2} .
$$

Corresponding inner product is given by

$$
\left\langle h_{1}, h_{2}\right\rangle_{S_{g}^{2}(\mathbb{D})}=\left\langle h_{1}, h_{2}\right\rangle+\left\langle D h_{1}, D h_{2}\right\rangle .
$$

In the following, we suppose that $g^{\prime}(z)$ has no zero points on $\overline{\mathbb{D}}$. This condition guarantees that $S_{g}^{2}(\mathbb{D})$ is closed under the given norm.

Theorem 1. Let $V_{g}$ be the weighted Volterra operator on $L_{a}^{2}(\mathbb{D})$. Then the following statements hold:

(i) Range of $V_{g}$ is $S_{g}^{2}(\mathbb{D})$.

(ii) $V_{g}$ is a bounded isomorphism from $L_{a}^{2}(\mathbb{D})$ to $S_{g}^{2}(\mathbb{D})$, and its inverse is $\left(1 / g^{\prime}(z)\right) D$.

(iii) The operator $T_{1}$ acting on $L_{a}^{2}(\mathbb{D})$ is similar under $V_{g}$ to the multiplication operator $M_{g}$ on $S_{g}^{2}(\mathbb{D})$.

Proof. (i) Let $h$ be in the range of $V_{g}$, and then there exists $f \in L_{a}^{2}(\mathbb{D})$ such that

$$
h(z)=\left(V_{g} f\right)(z)=\int_{0}^{z} g^{\prime}(w) f(w) d w .
$$


So $D h(z)=g^{\prime}(z) f(z), h(0)=0$, and $D h(z) / g^{\prime}(z) \in L_{a}^{2}(\mathbb{D})$. Therefore, $h(z) \in S_{g}^{2}(\mathbb{D})$. Conversely, suppose that $h \in S_{g}^{2}(\mathbb{D})$; then

$$
\begin{aligned}
V_{g}\left(\frac{D h}{g^{\prime}(z)}\right)(z) & =\int_{0}^{z} g^{\prime}(w) \frac{D h}{g^{\prime}(w)}(w) d w \\
& =h(z)-h(0)=h(z) .
\end{aligned}
$$

Hence $h$ belongs to the range of $V_{g}$.

(ii) First we want to show $V_{g}$ is a bounded operator on $L_{a}^{2}(\mathbb{D})$. For $f \in L_{a}^{2}(\mathbb{D})$, we have

$$
\begin{aligned}
\left\|V_{g} f\right\|_{S_{g}^{2}(\mathbb{D})}^{2} & =\left\|V_{g} f\right\|_{2}^{2}+\left\|D\left(V_{g} f\right)\right\|_{2}^{2} \leq 2\left\|D\left(V_{g} f\right)\right\|_{2}^{2} \\
& \leq 2\left\|g^{\prime}(z)\right\|_{\infty}^{2}\|f\|_{2}^{2} .
\end{aligned}
$$

Clearly $V_{g}$ is linear. To show $V_{g}$ is one to one, suppose that $f_{1}, f_{2} \in L_{a}^{2}(\mathbb{D})$, satisfying that

$$
\int_{0}^{z} g^{\prime}(w) f_{1}(w) d w=\int_{0}^{z} g^{\prime}(w) f_{2}(w) d w
$$

$\forall z \in \mathbb{D}$.

Differentiating both sides, we obtain that $f_{1}(z)=f_{2}(z)$ and hence $V_{g}$ is one to one. From the definition of $V_{g}$ we have that $V_{g}\left(\left(1 / g^{\prime}(z)\right) D h\right)(z)=h(z)$, for $h \in S_{g}^{2}(\mathbb{D})$, and $\left(1 / g^{\prime}(z)\right) D\left(V_{g} f\right)(z)=f(z)$, for $f \in L_{a}^{2}(\mathbb{D})$.

Therefore, $V_{g}$ is a bounded bijective linear operator from $L_{a}^{2}(\mathbb{D})$ onto $S_{g}^{2}(\mathbb{D})$, and $V_{g}^{-1}=\left(1 / g^{\prime}\right) D$.

(iii) Suppose $f$ belongs to $L_{a}^{2}(\mathbb{D})$, and $\left(V_{g} f\right)(z)=h(z)$. Note that

$$
\begin{aligned}
\left(T_{1} f\right)(z) & =\left(M_{g} f\right)(z)+\left(V_{g} f\right)(z) \\
& =g(z) \frac{h^{\prime}(z)}{g^{\prime}(z)}+h(z) .
\end{aligned}
$$

Now applying $V_{g}$ on both sides of the above equality, we obtain that

$$
\begin{aligned}
\left(V_{g} T_{1} f\right)(z) & =\int_{0}^{z} g^{\prime}(w)\left[\frac{h^{\prime}(w)}{g^{\prime}(w)} g(w)+h(w)\right] d w \\
& =\int_{0}^{z}\left[g(w) h^{\prime}(w)+g^{\prime}(w) h(w)\right] d w \\
& =\int_{0}^{z} D(g(w) h(w)) d w=g(z) h(z) \\
& =M_{g}\left(V_{g} f\right)(z) .
\end{aligned}
$$

So $V_{g} T_{1}=M_{g} V_{g}$ and $V_{g} T_{1} V_{g}^{-1}=M_{g}$. That is to say, $V_{g}$ transforms the operator $T_{1}$ into the multiplication operator $M_{g}$ on $S_{g}^{2}(\mathbb{D})$.

\section{The Reducing Subspaces of the Operator $T_{2}$}

In this section, for fixed $n \geq 2$, we consider the case of $g(z)=$ $z^{n}$. That is, the operator $T_{2}$ is defined by

$$
\begin{aligned}
\left(T_{2} f\right)(z) & =M_{z^{n}} f(z)+\left(V_{z^{n}} f\right)(z) \\
& =M_{z^{n}} f(z)+\int_{0}^{z} n w^{n-1} f(w) d w,
\end{aligned}
$$

$$
\text { for } f \in L_{a}^{2}(\mathbb{D}) \text {. }
$$

Since the $n$-shift operator is a contraction operator, and the operator $V_{z^{n}}$ is a bounded operators, $T_{2}$ is a bounded operator on $L_{a}^{2}(\mathbb{D})$.

Let $\mathscr{A}^{\prime}(T)$ denote the commutant of $T$, that is, $\mathscr{A}^{\prime}(T)=$ $\{S \in L(H) \mid T S=S T\}$, where $L(H)$ represents the collection of all bounded linear operators on a Hilbert space $H$. Then we have the following lemma.

Lemma 2. Let $L_{a}^{2}(\mathbb{D})$ be the Bergman space. If $P$ is a bounded operator on $L_{a}^{2}(\mathbb{D})$, then $P \in \mathscr{A}^{\prime}\left(M_{z}\right)$ if and only if $P$ admits the following matrix representation:

$$
P
$$

$$
=\left(\begin{array}{cccccc}
p_{11} & 0 & 0 & \cdots & 0 & \cdots \\
p_{21} & p_{11} & 0 & \cdots & 0 & \cdots \\
p_{31} & \frac{\gamma_{1}}{\gamma_{0}} \frac{\gamma_{1}}{\gamma_{2}} p_{21} & p_{11} & \cdots & 0 & \cdots \\
\vdots & \vdots & \vdots & \ddots & \vdots & \cdots \\
p_{k 1} & \frac{\gamma_{1}}{\gamma_{0}} \frac{\gamma_{k-2}}{\gamma_{k-1}} p_{k-1,1} & \frac{\gamma_{2}}{\gamma_{0}} \frac{\gamma_{k-3}}{\gamma_{k-1}} p_{k-2,1} & \cdots & p_{11} & \cdots \\
\vdots & \vdots & \vdots & \vdots & \vdots & \ddots
\end{array}\right)
$$

with respect to the orthonormal basis $\left\{e_{k}(z)=\gamma_{k} z^{k}\right\}_{k=0}^{\infty}$ of $L_{a}^{2}(\mathbb{D})$, where $p_{j k} \in \mathbb{C}(j, k \geq 1)$ and $\gamma_{k}=\sqrt{k+1}$. Moreover, if $P$ is a projection, then $P \in \mathscr{A}^{\prime}\left(M_{z}\right)$ if and only if $P=I$ or 0 .

Proof. Denote the orthonormal basis of $L_{a}^{2}(\mathbb{D})$ by $\left\{e_{k}(z)=\right.$ $\left.\gamma_{k} z^{k}\right\}_{k=0}^{\infty}$. Note that

$$
M_{z} e_{k}=z \gamma_{k} z^{k}=\frac{\gamma_{k}}{\gamma_{k+1}} e_{k+1} .
$$

So the operator $M_{z}$ admits the following matrix representation with respect to the above basis:

$$
M_{z}=\left(\begin{array}{cccccc}
0 & 0 & \cdots & 0 & 0 & \cdots \\
\frac{\gamma_{0}}{\gamma_{1}} & 0 & \cdots & 0 & 0 & \cdots \\
0 & \frac{\gamma_{1}}{\gamma_{2}} & \cdots & 0 & 0 & \cdots \\
\vdots & \vdots & \vdots & \vdots & \vdots & \cdots \\
0 & 0 & \cdots & \frac{\gamma_{k-1}}{\gamma_{k}} & 0 & \cdots \\
\vdots & \vdots & \vdots & \vdots & \ddots & \ddots
\end{array}\right) .
$$


Suppose that $P$ has the following matrix representation with respect to the orthonormal basis of $L_{a}^{2}(\mathbb{D})$ :

$$
P=\left(\begin{array}{cccccc}
p_{11} & p_{12} & p_{13} & \cdots & p_{1 k} & \cdots \\
p_{21} & p_{22} & p_{23} & \cdots & p_{2 k} & \cdots \\
p_{31} & p_{32} & p_{33} & \cdots & p_{3 k} & \cdots \\
\vdots & \vdots & \vdots & \vdots & \vdots & \\
p_{k 1} & p_{k 2} & p_{k 3} & \cdots & p_{k k} & \cdots \\
\vdots & \vdots & \vdots & \vdots & \vdots & \ddots
\end{array}\right)
$$

From $M_{z} P=P M_{z}$, we have

$$
\left.\begin{array}{c}
\left(\begin{array}{ccccc}
0 & 0 & \cdots & 0 & \cdots \\
\frac{\gamma_{0}}{\gamma_{1}} p_{11} & \frac{\gamma_{0}}{\gamma_{1}} p_{12} & \cdots & \frac{\gamma_{0}}{\gamma_{1}} p_{1 k} & \cdots \\
\frac{\gamma_{1}}{\gamma_{2}} p_{21} & \frac{\gamma_{1}}{\gamma_{2}} p_{22} & \cdots & \frac{\gamma_{1}}{\gamma_{2}} p_{2 k} & \cdots \\
\vdots & \vdots & \vdots & \vdots & \\
\frac{\gamma_{k-2}}{\gamma_{k-1}} p_{k-1,1} & \frac{\gamma_{k-2}}{\gamma_{k-1}} p_{k-1,2} & \cdots & \frac{\gamma_{k-2}}{\gamma_{k-1}} p_{k-1, k} & \cdots \\
\vdots & \vdots & \vdots & \vdots & \ddots
\end{array}\right) \\
\left(\begin{array}{ccccc}
\frac{\gamma_{0}}{\gamma_{1}} p_{12} & \frac{\gamma_{1}}{\gamma_{2}} p_{13} & \cdots & \frac{\gamma_{k-2}}{\gamma_{k-1}} p_{1 k} & \cdots \\
\frac{\gamma_{0}}{\gamma_{1}} p_{22} & \frac{\gamma_{1}}{\gamma_{2}} p_{23} & \cdots & \frac{\gamma_{k-2}}{\gamma_{k-1}} p_{2 k} & \cdots \\
\vdots & \vdots & \vdots & \vdots & \cdots \\
\frac{\gamma_{0}}{\gamma_{1}} p_{k 2} & \frac{\gamma_{1}}{\gamma_{2}} p_{k 3} & \cdots & \frac{\gamma_{k-2}}{\gamma_{k-1}} p_{k k} & \cdots \\
\vdots & \vdots & \vdots & \vdots & \ddots
\end{array}\right)
\end{array}\right)
$$

Thus

$$
\begin{aligned}
p_{i j} & =0, \quad i<j, \\
p_{i i} & =p_{11}, \quad i=2,3, \ldots, \\
\frac{\gamma_{j+k-1}}{\gamma_{j-1}} p_{j+k, j} & =\frac{\gamma_{j+k-2}}{\gamma_{j-2}} p_{j+k-1, j-1}, \quad(j \geq 2, \quad k \geq 1) .
\end{aligned}
$$

So we obtain

P

$$
=\left(\begin{array}{cccccc}
p_{11} & 0 & 0 & \cdots & 0 & \cdots \\
p_{21} & p_{11} & 0 & \cdots & 0 & \cdots \\
p_{31} & \frac{\gamma_{1}}{\gamma_{0}} \frac{\gamma_{1}}{\gamma_{2}} p_{21} & p_{11} & \cdots & 0 & \cdots \\
\vdots & \vdots & \vdots & \ddots & \vdots & \cdots \\
p_{k 1} & \frac{\gamma_{1}}{\gamma_{0}} \frac{\gamma_{k-2}}{\gamma_{k-1}} p_{k-1,1} & \frac{\gamma_{2}}{\gamma_{0}} \frac{\gamma_{k-3}}{\gamma_{k-1}} p_{k-2,1} & \cdots & p_{11} & \cdots \\
\vdots & \vdots & \vdots & \vdots & \vdots & \ddots
\end{array}\right) .
$$

Conversely, if $P$ admits the matrix representation (30) with respect to the above basis, simple computation shows that $M_{z} P=P M_{z}$. So $P \in \mathscr{A}^{\prime}\left(M_{z}\right)$. Moreover, if $P$ is a projection, we deduce that $P \in \mathscr{A}^{\prime}\left(M_{z}\right)$ if and only if $P$ has the following form:

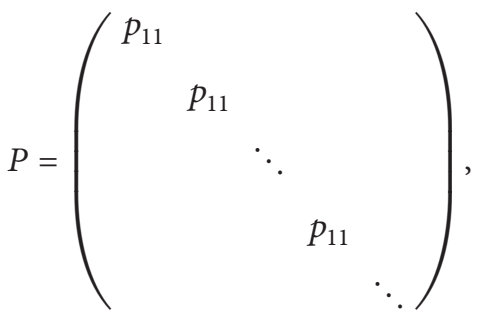

where $p_{11}=1$ or 0 .

Remark 3. In fact, since $M_{z}$ is irreducible on $L_{a}^{2}(\mathbb{D})$, any projection in $\mathscr{A}^{\prime}\left(M_{z}\right)$ is $I$ or 0 .

The following lemma will be used in the proof of main theorem.

Lemma 4. Let $H_{j}=\overline{\operatorname{span}}\left\{e_{n k+j}: k \geq 0\right\}(j=0,1, \ldots, n-1)$. Then

(i) $\left\{e_{n k+j}\right\}_{k=0}^{\infty}$ forms an orthonormal basis of $H_{j}$.

(ii) $L_{a}^{2}(\mathbb{D})=H_{0} \oplus H_{1} \oplus \cdots \oplus H_{n-1}$.

(iii) $H_{j}$ is a reducing subspace of $T_{2}$.

Proof. (i) and (ii) are obvious. We only need to show (iii). Note that

$$
\begin{aligned}
T_{2} e_{n k+j}(z) & =M_{z^{n}} e_{n k+j}(z)+\left(V_{z^{n}}\right) e_{n k+j}(z) \\
& =z^{n} \gamma_{n k+j} z^{n k+j}+\int_{0}^{z} n w^{n-1} \gamma_{n k+j} w^{n k+j} d w \\
& =\left(1+\frac{n}{n(k+1)+j}\right) \frac{\gamma_{n k+j}}{\gamma_{n(k+1)+j}} e_{n(k+1)+j} .
\end{aligned}
$$

So we have $T_{2} H_{j} \subset H_{j}$ and

$$
\begin{gathered}
T_{2}\left(H_{0} \oplus H_{1} \oplus \cdots \oplus H_{j-1} \oplus H_{j+1} \oplus \cdots \oplus H_{n-1}\right) \\
\subset H_{0} \oplus H_{1} \oplus \cdots \oplus H_{j-1} \oplus H_{j+1} \oplus \cdots \oplus H_{n-1},
\end{gathered}
$$

as desired. 
Set $T_{2 j}=\left.T_{2}\right|_{H_{j}}(j=0,1, \ldots, n-1)$. Then we have the following theorem.

Theorem 5. If $Q: L_{a}^{2}(\mathbb{D}) \rightarrow L_{a}^{2}(\mathbb{D})$ is a projection, then

$$
\begin{gathered}
Q\left(\begin{array}{llll}
T_{20} & & & \\
& T_{21} & & \\
& & \ddots & \\
& & & T_{2, n-1}
\end{array}\right) \\
=\left(\begin{array}{llll}
T_{20} & & \\
& T_{21} & \\
& & \ddots & \\
& & & T_{2, n-1}
\end{array}\right) Q
\end{gathered}
$$

if and only if

$$
Q=\left(\begin{array}{llll}
Q_{0} & & & \\
& Q_{1} & & \\
& & \ddots & \\
& & & Q_{n-1}
\end{array}\right),
$$

where $Q_{i}(i=0,1, \ldots, n-1)$ is $I_{H_{i}}$ or 0 .

Proof. If $Q \in \mathscr{A}^{\prime}\left(\operatorname{diag}\left(T_{20}, T_{21}, \ldots, T_{2, n-1}\right)\right)$, note that $L_{a}^{2}(\mathbb{D})=$ $H_{0} \oplus H_{1} \oplus \cdots \oplus H_{n-1}$, and then the operator $Q$ can be decomposed in the following form:

$$
Q=\left(\begin{array}{cccc}
Q_{00} & Q_{01} & \cdots & Q_{0, n-1} \\
Q_{10} & Q_{11} & \cdots & Q_{1, n-1} \\
\vdots & \vdots & \vdots & \vdots \\
Q_{n-1,0} & Q_{n-1,1} & \cdots & Q_{n-1, n-1}
\end{array}\right)
$$

$$
T_{2 j}=\left(\begin{array}{cccccc}
0 & 0 & \cdots & 0 & 0 & \cdots \\
\left(1+\frac{n}{n+j}\right) \frac{\gamma_{j}}{\gamma_{n+j}} & 0 & \cdots & 0 & 0 & \cdots \\
0 & \left(1+\frac{n}{2 n+j}\right) \frac{\gamma_{n+j}}{\gamma_{2 n+j}} & \cdots & 0 & 0 & \cdots \\
\vdots & \vdots & \vdots & \vdots & \vdots & \cdots \\
0 & 0 & \cdots & \left(1+\frac{n}{n(k+1)+j}\right) \frac{\gamma_{n k+j}}{\gamma_{n(k+1)+j}} & 0 & \cdots \\
\vdots & \vdots & \vdots & \ddots & \ddots
\end{array}\right) .
$$

Case $1(i=j)$. From (37), we get $Q_{j j} T_{2 j}=T_{2 j} Q_{j j}(j=$ $0,1, \ldots, n-1)$. Applying Lemma 2 , we know $Q_{j j}$ has the form where $Q_{i j}: H_{j} \rightarrow H_{i}(i, j=0,1, \ldots, n-1)$. The condition $Q \in \mathscr{A}^{\prime}\left(\operatorname{diag}\left(T_{20}, T_{21}, \ldots, T_{2, n-1}\right)\right)$ yields that

$$
\begin{gathered}
\left(\begin{array}{cccc}
Q_{00} T_{20} & Q_{01} T_{21} & \cdots & Q_{0, n-1} T_{2, n-1} \\
Q_{10} T_{20} & Q_{11} T_{21} & \cdots & Q_{1, n-1} T_{2, n-1} \\
\vdots & \vdots & \vdots & \vdots \\
Q_{n-1,0} T_{20} & Q_{n-1,1} T_{21} & \cdots & Q_{n-1, n-1} T_{2, n-1}
\end{array}\right) \\
=\left(\begin{array}{cccc}
T_{20} Q_{00} & T_{20} Q_{01} & \cdots & T_{20} Q_{0, n-1} \\
T_{21} Q_{10} & T_{21} Q_{11} & \cdots & T_{21} Q_{1, n-1} \\
\vdots & \vdots & \vdots & \vdots \\
T_{2, n-1} Q_{n-1,0} & T_{2, n-1} Q_{n-1,1} & \cdots & T_{2, n-1} Q_{n-1, n-1}
\end{array}\right) .
\end{gathered}
$$

Suppose that $Q_{i j}$ has the following matrix representation with respect to the orthonormal basis of $H_{j}$;

$$
Q_{i j}=\left(\begin{array}{cccccc}
q_{11}^{i j} & q_{12}^{i j} & q_{13}^{i j} & \cdots & q_{1 k}^{i j} & \cdots \\
q_{21}^{i j} & q_{22}^{i j} & q_{23}^{i j} & \cdots & q_{2 k}^{i j} & \cdots \\
q_{31}^{i j} & q_{32}^{i j} & q_{33}^{i j} & \cdots & q_{3 k}^{i j} & \cdots \\
\vdots & \vdots & \vdots & \vdots & \vdots & \\
q_{k 1}^{i j} & q_{k 2}^{i j} & q_{k 3}^{i j} & \cdots & q_{k k}^{i j} & \cdots \\
\vdots & \vdots & \vdots & \vdots & \vdots & \ddots
\end{array}\right),
$$

where $q_{t_{1}+1, t_{2}+1}^{i j}=\left\langle Q_{i j} e_{t_{2} n+j}, e_{t_{1} n+i}\right\rangle\left(t_{1}, t_{2}=0,1, \ldots\right)$.

From (32), we know the operator $T_{2 j}$ admits the following matrix representation with respect to the basis of $H_{j}$;

similar to (30). Note that $Q$ is a projection, so $Q_{j j}=Q_{j j}^{*}$. Hence $Q_{j j}=q_{11}^{j j} I_{H_{j}}\left(q_{11}^{j j} \in \mathbb{R}\right)$. 
Case $2(i \neq j)$. From (37), we have that $T_{2 i} Q_{i j}=Q_{i j} T_{2 j}$. This equality is equivalent to

$$
\begin{aligned}
& \left(\begin{array}{cccc}
0 & 0 & 0 \\
\left(1+\frac{n}{n+i}\right) \frac{\gamma_{i}}{\gamma_{n+i}} q_{11}^{i j} & \left(1+\frac{n}{n+i}\right) \frac{\gamma_{i}}{\gamma_{n+i}} q_{12}^{i j} & \cdot & \left(1+\frac{n}{n+i}\right) \frac{\gamma_{i}}{\gamma_{n+i}} q_{1 k}^{i j} \\
\left(1+\frac{n}{2 n+i}\right) \frac{\gamma_{n+i}}{\gamma_{2 n+i}} q_{21}^{i j} & \left(1+\frac{n}{2 n+i}\right) \frac{\gamma_{n+i}}{\gamma_{2 n+i}} q_{22}^{i j} & \cdot & \left(1+\frac{n}{2 n+i}\right) \frac{\gamma_{n+i}}{\gamma_{2 n+i}} q_{2 k}^{i j} \\
\cdot & \cdot & \cdot \\
\left(1+\frac{n}{(k-1) n+i}\right) \frac{\gamma_{(k-2) n+i}}{\gamma_{(k-1) n+i}} q_{k-1,1}^{i j} & \left(1+\frac{n}{(k-1) n+i}\right) \frac{\gamma_{(k-2) n+i}}{\gamma_{(k-1) n+i}} q_{k-1,2}^{i j} & \cdot & \left(1+\frac{n}{(k-1) n+i}\right) \frac{\gamma_{(k-2) n+i}}{\gamma_{(k-1) n+i}} q_{k-1, k}^{i j}
\end{array}\right)
\end{aligned}
$$

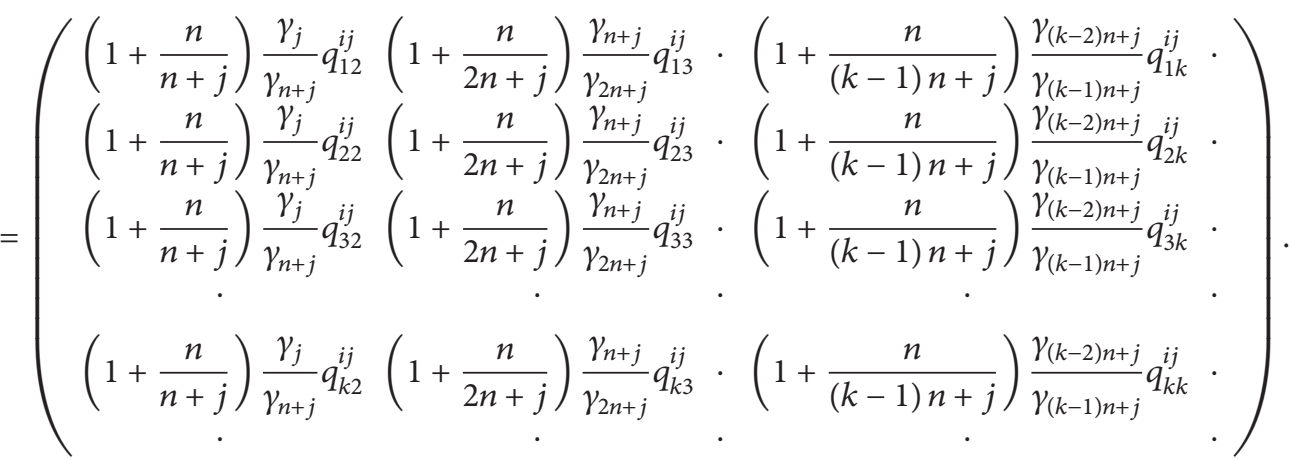

So we obtain

$$
Q_{i j}=\left(\begin{array}{cccccc}
q_{11}^{i j} & 0 & 0 & 0 & \cdots \\
q_{21}^{i j} & \frac{1+n /(n+i)}{1+n /(n+j)} \frac{\gamma_{i}}{\gamma_{j}} \frac{\gamma_{n+j}}{\gamma_{n+i}} q_{11}^{i j} & 0 & 0 & \cdots \\
q_{31}^{i j} & \frac{1+n /(2 n+i)}{1+n /(n+j)} \frac{\gamma_{n+j}}{\gamma_{j}} \frac{\gamma_{n+i}}{\gamma_{2 n+i}} q_{21}^{i j} & \frac{1+n /(n+i)}{1+n /(n+j)} \frac{1+n /(2 n+i)}{1+n /(2 n+j)} \frac{\gamma_{i}}{\gamma_{j}} \frac{\gamma_{2 n+j}}{\gamma_{2 n+i}} q_{11}^{i j} & 0 & \cdots \\
\vdots & \vdots & \vdots & \vdots & \vdots
\end{array}\right) .
$$

On the other hand, from (37) we also have $T_{2 j} Q_{j i}=Q_{j i} T_{2 i}$.

In the same way as for $T_{2 i} Q_{i j}=Q_{i j} T_{2 j}$, we get

$$
Q_{j i}=\left(\begin{array}{ccccc}
q_{11}^{j i} & 0 & 0 & 0 & \cdots \\
q_{21}^{j i} & \frac{1+n /(n+j)}{1+n /(n+i)} \frac{\gamma_{j}}{\gamma_{i}} \frac{\gamma_{n+i}}{\gamma_{n+j}} q_{11}^{j i} & 0 & 0 & \cdots \\
q_{31}^{j i} & \frac{1+n /(2 n+j)}{1+n /(n+i)} \frac{\gamma_{n+i}}{\gamma_{i}} \frac{\gamma_{n+j}}{\gamma_{2 n+j}} q_{21}^{j i} & \frac{1+n /(n+j)}{1+n /(n+i)} \frac{1+n /(2 n+j)}{1+n /(2 n+i)} \frac{\gamma_{j}}{\gamma_{i}} \frac{\gamma_{2 n+i}}{\gamma_{2 n+j}} q_{11}^{j i} & 0 & \cdots \\
\vdots & \vdots & \vdots & \vdots & \vdots
\end{array}\right)
$$


$Q$ is a projection that yields $Q_{i j}^{*}=Q_{j i}$. Thus we have $q_{l 1}^{i j}=$ $0(l=2,3, \ldots)$. Solving the system of equations

$$
\begin{aligned}
\bar{q}_{11}^{i j} & =q_{11}^{j i}, \\
\frac{1+n /(n+i)}{1+n /(n+j)} \frac{\gamma_{i}}{\gamma_{j}} \frac{\gamma_{n+j}}{\gamma_{n+i}} \bar{q}_{11}^{i j} & =\frac{1+n /(n+j)}{1+n /(n+i)} \frac{\gamma_{j}}{\gamma_{i}} \frac{\gamma_{n+i}}{\gamma_{n+j}} q_{11}^{j i},
\end{aligned}
$$

we get $q_{11}^{i j}=0$. Therefore, $Q_{i j}=0 . Q^{2}=Q$ implies that

$$
Q=\left(\begin{array}{cccc}
Q_{0} & & & \\
& Q_{1} & & \\
& & \ddots & \\
& & & Q_{n-1}
\end{array}\right),
$$

where $Q_{i}(i=0,1, \ldots, n-1)$ is $I_{H_{i}}$ or 0 .

Conversely, if

$$
Q=\left(\begin{array}{cccc}
Q_{0} & & & \\
& Q_{1} & & \\
& & \ddots & \\
& & & Q_{n-1}
\end{array}\right),
$$

where $Q_{i}(i=0,1, \ldots, n-1)$ is $I_{H_{i}}$ or 0 , it is obvious that $Q \in \mathscr{A}^{\prime}\left(\operatorname{diag}\left(T_{20}, T_{21}, \ldots, T_{2, n-1}\right)\right)$.

From [14], we know that determining the reducing subspaces of $T_{2}$ is equivalent to finding the projection in the commutant of $T_{2}$. Thus we have the following conclusion.

Theorem 6 (main theorem). Let $L_{a}^{2}(\mathbb{D})$ be the Bergman space. For $n \geq 2$, the operator $T_{2}$ has $2^{n}$ reducing subspaces with minimal reducing subspaces $H_{0}, H_{1}, \ldots, H_{n-1}$.

Proof. Suppose that $Q T_{2}=T_{2} Q$ for a projection $Q$. Note that $\left.T_{2}\right|_{L_{a}^{2}(\mathbb{D})}=T_{20} \oplus T_{21} \oplus \cdots \oplus T_{2, n-1}$. Hence

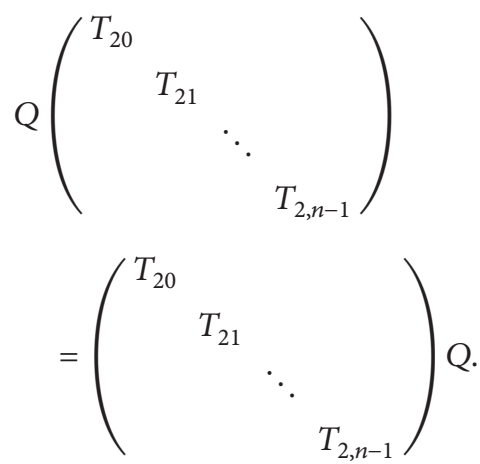

By Theorem 5, the projection operator

$$
Q=\left(\begin{array}{cccc}
Q_{0} & & & \\
& Q_{1} & & \\
& & \ddots & \\
& & & Q_{n-1}
\end{array}\right) \text {, }
$$

where $Q_{i}(i=0,1, \ldots, n-1)$ is $I_{H_{i}}$ or 0 . Applying Lemma 4, we have that the reducing subspaces of $T_{2}$ are

$$
\begin{aligned}
& c_{0} H_{0} \oplus c_{1} H_{1} \oplus \cdots \oplus c_{n-1} H_{n-1}, \\
& \text { where } c_{i}=0 \text { or } 1(i=0,1, \ldots, n-1),
\end{aligned}
$$

and the minimal reducing subspaces are $H_{0}, H_{1}, \ldots, H_{n-1}$.

\section{Some Consequences}

In this section, we use the characterization of the reducing subspaces of $T_{2}$ to obtain a description of reducing subspaces of $M_{z^{n}}$ on $S_{n}^{2}(\mathbb{D})$, which is similar to $T_{2}$.

The space $S_{n}^{2}(\mathbb{D})$ is defined by

$$
\begin{aligned}
& S_{n}^{2}(\mathbb{D})=\left\{f \in H(\mathbb{D}): D f \in L_{a}^{2}(\mathbb{D}), f^{(l)}(0)=0, l\right. \\
& \quad=0,1, \ldots, n-1\} .
\end{aligned}
$$

If $f(z) \in S_{n}^{2}(\mathbb{D})$, it follows that $f(z)=\sum_{k=0}^{\infty} a_{k} z^{n+k}$, and then $D f(z)=\sum_{k=0}^{\infty}(n+k) a_{k} z^{n+k-1}$. Since $D f \in L_{a}^{2}(\mathbb{D})$, we have $\|D f(z)\|_{2}^{2}=\sum_{k=0}^{\infty}(n+k)\left|a_{k}\right|^{2}$, and

$$
\begin{aligned}
\int_{\mathbb{D}}|f(z)|^{2} d A(z) & =\sum_{k=0}^{\infty} \frac{\left|a_{k}\right|^{2}}{n+k+1} \leq \sum_{k=0}^{\infty}(n+k)\left|a_{k}\right|^{2} \\
& =\|D f(z)\|_{2}^{2} .
\end{aligned}
$$

So $f(z) \in L_{a}^{2}(\mathbb{D})$. The norm of $S_{n}^{2}(\mathbb{D})$ is defined by

$$
\|f\|_{S_{n}^{2}(\mathbb{D})}^{2}=\|f\|_{2}^{2}+\|D f\|_{2}^{2} .
$$

Corresponding inner product is given by

$$
\langle f, g\rangle_{S_{n}^{2}(\mathbb{D})}=\langle f, g\rangle+\langle D f, D g\rangle .
$$

The proof of the following theorem is similar to the proof of Theorem 1, so we omit it.

Theorem 7. Let $V_{z^{n}}$ be the weighted Volterra operator on $L_{a}^{2}(\mathbb{D})$. Then the following statements hold:

(i) Range of $V_{z^{n}}$ is $S_{n}^{2}(\mathbb{D})$.

(ii) $V_{z^{n}}$ is a bounded isomorphism from $L_{a}^{2}(\mathbb{D})$ to $S_{n}^{2}(\mathbb{D})$, and its inverse is $\left(1 / n z^{n-1}\right) D$. (Remark: Note $\left(1 / n z^{n-1}\right) D$ acting on $S_{n}^{2}(\mathbb{D})$, and from the definition of $S_{n}^{2}(\mathbb{D})$, we know that $z=0$ is a removable singular point of $\left(1 / n z^{n-1}\right) D g(z)$, for $g(z) \in S_{n}^{2}(\mathbb{D})$. We can define $\left.\left(1 / n z^{n-1}\right) D g(z)\right|_{z=0}$ by $\lim _{z \rightarrow 0}\left(1 / n z^{n-1}\right) \operatorname{Dg}(z)$. Then $z=0$ can be viewed as an analytic point of $\left.\left(1 / n z^{n-1}\right) D g(z)\right)$.

(iii) The operator $T_{2}$ acting on $L_{a}^{2}(\mathbb{D})$ is similar under $V_{z^{n}}$ to the multiplication operator $M_{z^{n}}$ on $S_{n}^{2}(\mathbb{D})$. 
Note that $S_{n}^{2}(\mathbb{D})=\overline{\operatorname{span}}\left\{z^{k+n}: k \geq 0\right\}$; from (52), we have

$$
\begin{aligned}
& \left\langle z^{k+n}, z^{m+n}\right\rangle_{S_{n}^{2}(\mathbb{D})} \\
& =\left\langle z^{k+n}, z^{m+n}\right\rangle+\left\langle(k+n) z^{k+n-1},(m+n) z^{m+n-1}\right\rangle \\
& = \begin{cases}\frac{(k+n)(k+n+1)+1}{k+n+1}, & k=m, \\
0, & k \neq m .\end{cases}
\end{aligned}
$$

Thus, the orthonormal basis of $S_{n}^{2}(\mathbb{D})$ is given by

$$
\left\{f_{k, n}(z)=\sqrt{\frac{k+n+1}{(k+n)(k+n+1)+1}} z^{k+n}\right\}_{k=0}^{\infty} .
$$

In order to characterize the reducing subspaces of $M_{z^{n}}$ on $S_{n}^{2}(\mathbb{D})$, we need the following lemma which is similar to Lemma 4.

Lemma 8. Let $L_{j}=\overline{\operatorname{span}}\left\{f_{n k+j, n}: k \geq 0\right\}(j=0,1, \ldots, n-1)$. Then

(i) $\left\{f_{n k+j, n}\right\}_{k=0}^{\infty}$ forms an orthonormal basis of $L_{j}$.

(ii) $S_{n}^{2}(\mathbb{D})=L_{0} \oplus L_{1} \oplus \cdots \oplus L_{n-1}$.

(iii) $L_{j}$ is a reducing subspace of $M_{z^{n}}$.

Corollary 9. Let $S_{n}^{2}(\mathbb{D})$ be described as the above. For $n \geq 2$, the multiplication operator $M_{z^{n}}$ has $2^{n}$ reducing subspaces with minimal reducing subspaces $L_{0}, L_{1}, \ldots, L_{n-1}$.

Proof. The proof of Corollary 9 is similar to Theorems 5 and 6 . Here we omit it.

Remark 10. In fact, $S_{n}^{2}(\mathbb{D})$ is a Hilbert space of type I which is considered by Stessin and Zhu in [12]. So Lemma 8 and Corollary 9 also follow from Stessin and Zhu's paper [12].

\section{Conflicts of Interest}

The authors declare that there are no conflicts of interest regarding the publication of this paper.

\section{Acknowledgments}

This research is supported by NNSF of China (11371119).

\section{References}

[1] A. Aleman, "A class of integral operators on spaces of analytic functions," in Topics in Complex Analysis and Operator Theory, pp. 3-30, University of Málaga, Málaga, Spain, 2007.

[2] A. Aleman and B. Korenblum, "Volterra invariant subspaces of $H^{p}$," Bulletin des Sciences Mathématiques, vol. 132, no. 6, pp. 510528, 2008.

[3] J. A. Ball, "Hardy space expectation operators and reducing subspaces," Proceedings of the American Mathematical Society, vol. 47, pp. 351-357, 1975.

[4] A. Beurling, "On two problems concerning linear transformations in Hilbert space," Acta Mathematica, vol. 81, 17 pages, 1948.
[5] C. C. Cowen, G. Gunatillake, and E. Ko, "Hermitian weighted composition operators and Bergman extremal functions," Complex Analysis and Operator Theory, vol. 7, no. 1, pp. 69-99, 2013.

[6] Ž. Čučković and B. Paudyal, "Invariant subspaces of the shift plus complex Volterra operator," Journal of Mathematical Analysis and Applications, vol. 426, no. 2, pp. 1174-1181, 2015.

[7] R. G. Douglas and Y.-S. Kim, "Reducing subspaces on the annulus," Integral Equations and Operator Theory, vol. 70, no. 1, pp. 1-15, 2011.

[8] B. I. Korenbljum, "Invariant subspaces of the shift operator in a weighted Hilbert space," Matematicheskii Sbornik, vol. 89, no. 131, pp. 110-137, 1972.

[9] A. Montes-Rodriguez, M. Ponce-Escudero, and S. A. Shkarin, "Invariant subspaces of parabolic self-maps in the Hardy space," Mathematical Research Letters, vol. 17, no. 1, pp. 99-107, 2010.

[10] E. A. Nordgren, "Reducing subspaces of analytic Toeplitz operators," Duke Mathematical Journal, vol. 34, pp. 175-181, 1967.

[11] D. Sarason, "A remark on the Volterra operator," Journal of Mathematical Analysis and Applications, vol. 12, pp. 244-246, 1965.

[12] M. Stessin and K. Zhu, "Reducing subspaces of weighted shift operators," Proceedings of the American Mathematical Society, vol. 130, no. 9, pp. 2631-2639, 2002.

[13] K. H. Zhu, "The reducing subspaces of a class of multiplication operator," Journal of the London Mathematical Society. Second Series, vol. 62, no. 2, pp. 553-568, 2000.

[14] R. G. Douglas, Banach Algebra Techniques in Operator Theory, Academic Press, New York, NY, USA, 1972.

[15] H. Hedenmalm, B. Korenblum, and K. Zhu, Theory of Bergman Spaces, vol. 199 of Graduate Texts in Mathematics, Springer, New York, NY, USA, 2000.

[16] Y. Katznelson, An Introduction to Harmonic Analysis, Cambridge Mathematical Library, Cambridge University Press, Cambridge, UK, 3rd edition, 2004.

[17] D. Sarason, "Invariant subspaces," in Topics in Operator Theory, vol. 13 of Mathematical Surveys, American Mathematical Society, Providence, RI, USA, 1974. 


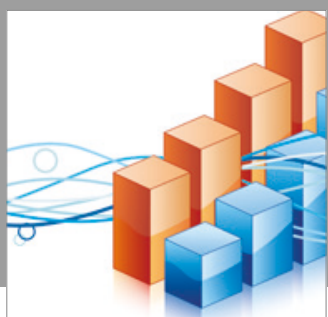

Advances in

Operations Research

vatersals

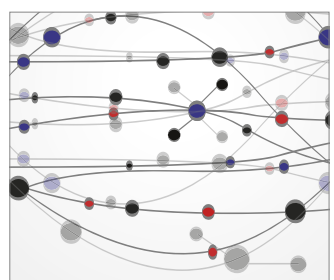

\section{The Scientific} World Journal
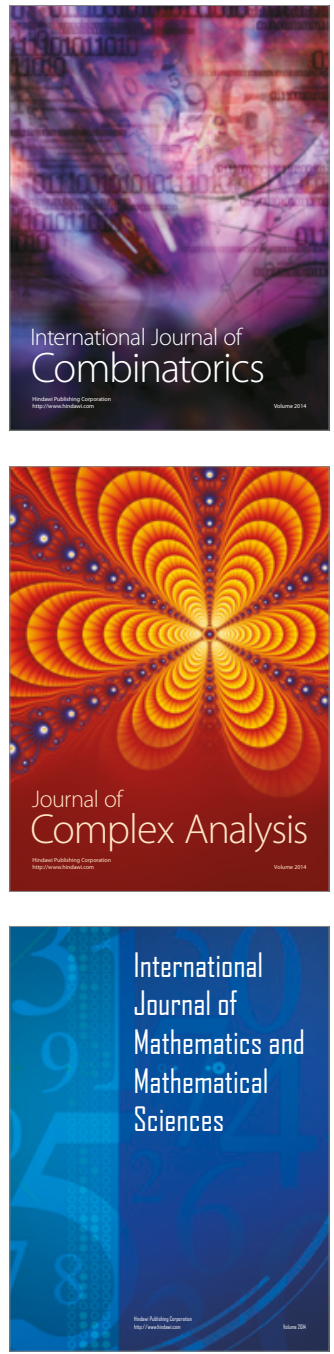
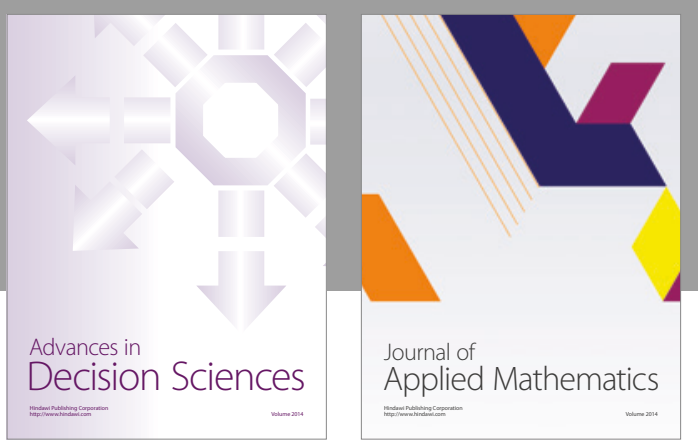

Algebra

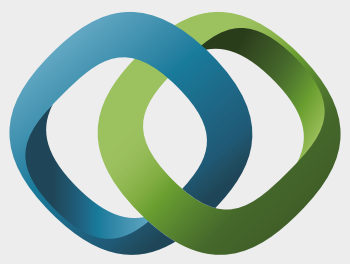

\section{Hindawi}

Submit your manuscripts at

https://www.hindawi.com
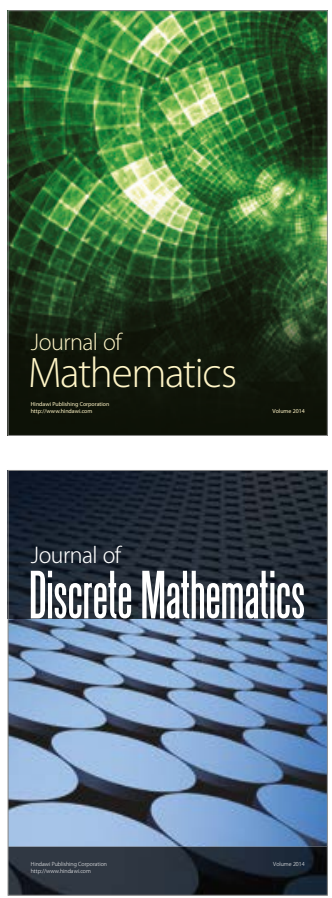

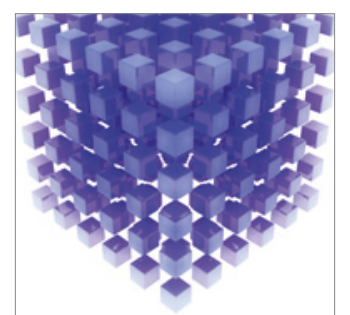

Mathematical Problems in Engineering
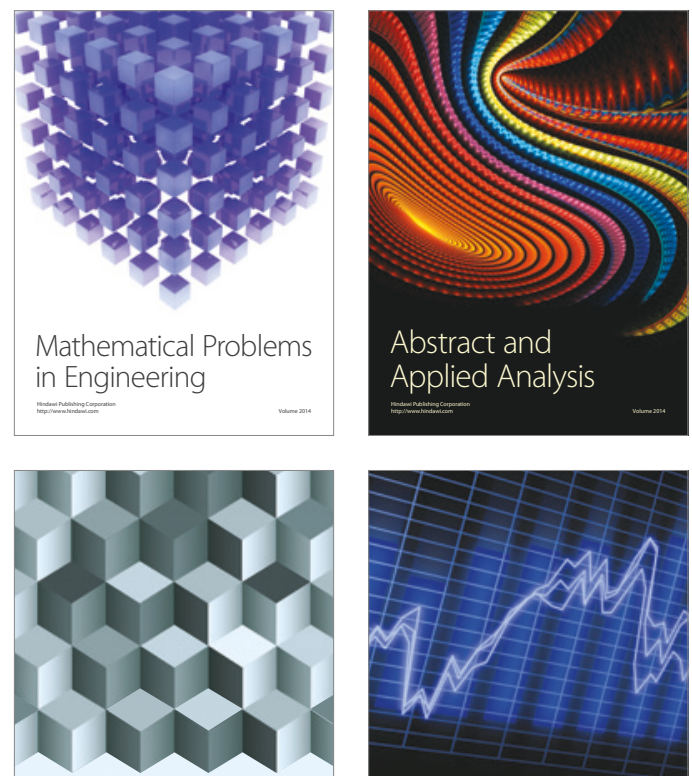

Journal of

Function Spaces

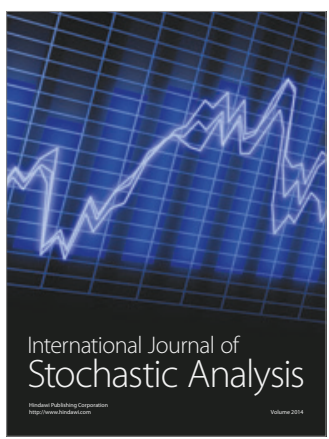

Probability and Statistics
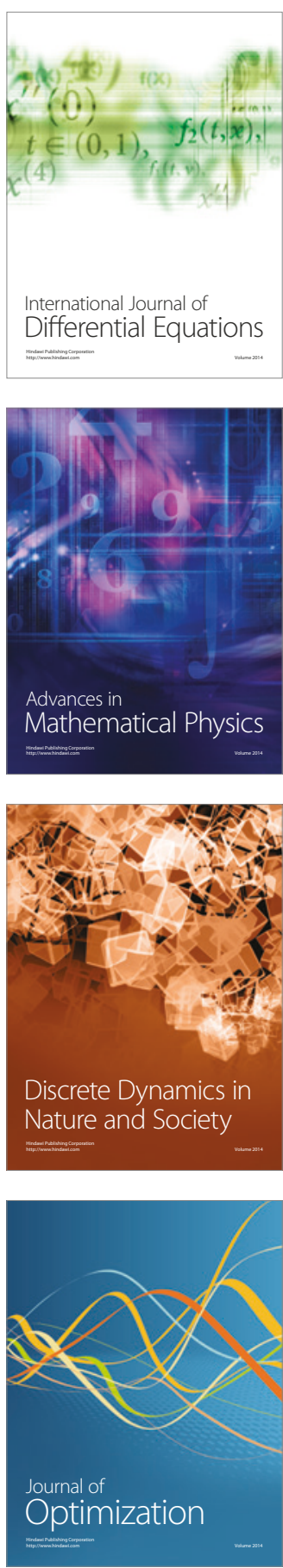